\title{
Abdominal Wound Dehiscence in Adults: Development and Validation of a Risk Model
}

\author{
Gabriëlle H. van Ramshorst · Jeroen Nieuwenhuizen • \\ Wim C. J. Hop · Pauline Arends · Johan Boom • \\ Johannes Jeekel $\cdot$ Johan F. Lange
}

Published online: 7 November 2009

(c) The Author(s) 2009. This article is published with open access at Springerlink.com

\begin{abstract}
Background Several studies have been performed to identify risk factors for abdominal wound dehiscence. No risk model had yet been developed for the general surgical population. The objective of the present study was to identify independent risk factors for abdominal wound dehiscence and to develop a risk model to recognize highrisk patients. Identification of high-risk patients offers opportunities for intervention strategies.

Methods Medical registers from January 1985 to December 2005 were searched. Patients who had primarily undergone appendectomies or nonsurgical (e.g., urological) operations were excluded. Each patient with abdominal wound dehiscence was matched with three controls by systematic random sampling. Putative relevant patientrelated, operation-related, and postoperative variables were evaluated in univariate analysis and subsequently entered in multivariate stepwise logistic regression models to delineate major independent predictors of abdominal wound dehiscence. A risk model was developed, which was validated in a population of patients who had undergone operation between January and December 2006.

Results A total of 363 cases and 1,089 controls were analyzed. Major independent risk factors were age, gender, chronic pulmonary disease, ascites, jaundice, anemia, emergency surgery, type of surgery, postoperative
\end{abstract}

G. H. van Ramshorst ( $₫)$. J. Nieuwenhuizen · P. Arends ·

J. Boom · J. Jeekel · J. F. Lange

Department of Surgery, Erasmus University Medical Center, Room Z-836, P.O. Box 2040, 3000 CA Rotterdam, Netherlands e-mail: g.vanramshorst@erasmusmc.nl

W. C. J. Hop

Department of Biostatistics, Erasmus University Medical Center, P.O. Box 2040, 3000 CA Rotterdam, Netherlands coughing, and wound infection. In the validation population, risk scores were significantly higher $(P<0.001)$ for patients with abdominal wound dehiscence $(n=19)$ compared to those without $(n=677)$. Resulting scores ranged from 0 to 8.5, and the risk for abdominal wound dehiscence over this range increased exponentially from $0.02 \%$ to $70.1 \%$.

Conclusions The validated risk model shows high predictive value for abdominal wound dehiscence and may help to identify patients at increased risk.

\section{Introduction}

Abdominal wound dehiscence (burst abdomen, fascial dehiscence) is a severe postoperative complication, with mortality rates reported as high as $45 \%$ [1-3]. The incidence, as described in the literature, ranges from $0.4 \%$ to $3.5 \%$ [417]. Abdominal wound dehiscence can result in evisceration, requiring immediate treatment. Prolonged hospital stay, high incidence of incisional hernia, and subsequent reoperations underline the severity of this complication.

Despite advances in perioperative care and suture materials, incidence and mortality rates in regard to abdominal wound dehiscence have not significantly changed over the past decades. This may be attributable to increasing incidences of risk factors within patient populations outweighing the benefits of technical achievements. Several mainly retrospective studies have been performed to identify risk factors for this complication, often presenting conflicting results. Unfortunately, multivariate analysis has only been performed in a minority of studies and in general on small numbers of patients [4-7, 10, 15].

The goal of the underlying study was to evaluate possible risk factors for abdominal wound dehiscence and to 
design a risk model based on independent risk factors. This model can be used to assess the risk for individual patients, and it may prove useful for prevention strategies in clinical studies, e.g., development of alternative closure techniques, in high-risk patients.

\section{Materials and methods}

All medical registers and operation records of adult patients from our academic teaching hospital dating from January 1985 to December 2005 were used for a computergenerated search of the keywords dehiscence, wound dehiscence, fascial dehiscence, and Platzbauch (widely used German term for abdominal wound dehiscence). Patients who had primarily undergone laparoscopic surgery, abdominal surgery in other wards (e.g., gynecology, urology), appendectomy, and umbilical and inguinal hernia surgery were excluded. Likewise, identified patients were excluded if insufficient evidence of fascial dehiscence (e.g., serous wound exudate production without confirmed fascial dehiscence) was found in clinical records.

For each case three suitable controls were randomly selected from a group of patients who had undergone open abdominal surgery as close as possible in time. For patients who had undergone operation on weekends and holidays, controls were selected from patients who had been operated between Sunday midnight and Friday midnight. This approach was chosen to avoid excessive inclusion of emergency operations in the control group, thereby ensuring that the control group is as representative for the "average" surgical population as possible. Controls were not matched according to age, sex, and type of surgery because these characteristics had been reported as risk factors in other studies and we intended to evaluate these factors as well. Moreover, patients who had undergone open abdomen treatment were excluded.

Patient and operation-related preoperative, perioperative, and postoperative variables and in-hospital mortality were recorded for all cases and controls by examining patient charts, operation records, laboratory and culture results, and discharge letters. Postoperative coughing was defined as coughing documented by doctors in the patient charts before the diagnosis of abdominal wound dehiscence, or before discharge in patients without abdominal wound dehiscence. Wound infection was defined as documented pus production, "infection" or "abscess" of the operative site prior to the diagnosis of abdominal wound dehiscence, or opening of the operative site on suspicion of infection without presence of negative wound cultures within 30 days after surgery. On the condition that at least $85 \%$ of data were complete, patients were compared with controls using the chi-square test or the Mann-Whitney
$U$-test for categorical or continuous data, respectively. Subsequently, multivariate stepwise logistic regression with backwards elimination was used to identify major independent predictors of abdominal wound dehiscence. The resulting regression coefficients for the major risk factors were used as weights for these variables to calculate a risk score for abdominal wound dehiscence.

All patients who had undergone open abdominal surgery between January and December 2006 were reviewed to validate the risk model. Medical registers were used to record the presence of risk factors for each patient, after which total scores were calculated and compared for patients with and without abdominal wound dehiscence. Patients were excluded for validation of the risk model if data on risk factors were absent. The goodness-of-fit of the risk model was assessed with the Hosmer and Lemeshow test. The predictive value of the risk model was assessed by plotting the sensitivity versus the fraction false-positives for all possible cut-off levels in a receiving operating characteristic curve (ROC curve). An area under the curve of 0.90 or greater is generally considered to denote high predictive value; $P$ values (two-sided) $<0.05$ were considered significant in all analyses.

\section{Results}

From January 1985 to December 2005, 429,906 operative procedures were performed at the department of surgery. The incidence of abdominal wound dehiscence did not show significant changes during the study period, and a total of 363 cases of abdominal wound dehiscence were identified and compared to 1,089 selected controls. Mean presentation of abdominal wound dehiscence was at postoperative day 9 (range: 0-32 days), with $90 \%$ of all cases presenting before the 15th postoperative day. Hospital stay was significantly longer $(P<0.001)$ for patients with abdominal wound dehiscence, with a median of 36 days, versus 16 days in the control group. In-hospital mortality for the two groups was $22 \%$ and $9 \%$, respectively $(P<0.001)$. Sixty-one patients were treated conservatively and 302 were treated operatively. Of these 302 patients, 29 developed recurrences of abdominal wound dehiscence within 30 days of reoperation $(9.3 \%)$, and 6 of them developed second recurrences. Women were treated conservatively more often than men $(P=0.03)$. Conservatively treated patients were comparable with operatively treated patients in terms of hospital stay [median 33 days versus 37 days $(P=0.339)]$, age $(P=0.379)$, mortality $(P=0.408)$, and comorbidity (all $P>0.05)$.

In most cases, tearing of sutures through the fascia was reported to be the cause of the dehiscence (29\%). Other reported causes were infection $(9 \%)$, broken suture $(8 \%)$, 
fascial necrosis (6\%), and loose knots (4\%). However, in $44 \%$ of all patients no explanation was recorded for abdominal wound dehiscence. Data were incomplete in more than $15 \%$ of subjects for smoking, body mass index (BMI), American Society of Anesthesiologists (ASA) class, hemodynamic instability, type of incision, and type of closure (such as continuous versus interrupted or type of suture used), or preoperative protein and albumin levels, which prevented us from entering these factors in univariate analysis.

The results of the univariate analyses are shown in Table 1. In the abdominal wound dehiscence group, the following variables were significantly more prevalent compared to the control group: old age, male gender, hypertension, chronic pulmonary disease, ascites, anemia, jaundice, corticosteroid use, sepsis, emergency surgery, postoperative coughing, wound infection (all $P<0.001$ ), uremia $(P=0.013$ ), and operative time $(P=0.003)$. Also, type of surgery differed between cases and controls. The subcategories "spleen" and "adrenal gland" were combined into the category "other" in view of the small group numbers. The variables diabetes mellitus, previous laparotomy, and postoperative vomiting were not found to be significant risk factors.

All variables that were significant in univariate analyses were entered in a multivariate stepwise logistic regression to determine which variables were significant independent risk factors (Table 2). In the evaluation of type of surgery, we expected the subcategory "abdominal wall" (including only "clean" operations, i.e., incisional hernia repair and exploratory laparotomy without further intervention) to be associated with the lowest risk of developing abdominal wound dehiscence. Therefore, this category was used as the reference category. For the variable "age," the reference category was patients under the age of 40 years. Adjusted for the significant risk factors, none of the other variables, including operative time, corticosteroid use, and sepsis, had significant effects.

Based on these findings, a risk model for abdominal wound dehiscence was developed. Because none of the surgery subcategories "liver," "kidney," or "pancreas" had proven significant risk factors, and because the effects of these variables did not significantly differ from one another $(P=0.81)$, regression coefficients were recalculated after combining these factors with "spleen" and "adrenal gland" in the category "other." For the subcategory "gallbladder and bile duct" a strong trend toward significance was found and led to the inclusion of this factor in the risk model. The risk scores, weighing the various factors by using the resulting regression coefficients in the logistic regression analysis, are shown in Table 3. If risk factors are absent (such as in a female patient or when another type of surgery is performed), no points are given. A higher value of the score predicts a higher risk.

\section{Validation of the risk model}

A total of 863 patients underwent open abdominal surgery between January and December 2006. Medical registers were used to record the presence of identified risk factors and abdominal wound dehiscence for every individual. In 177 cases, including 3 cases of abdominal wound dehiscence, data on one or more major risk factors were missing, leaving 686 cases for validation of the risk model. The incidence of abdominal wound dehiscence in this group was $2.8 \%$ (19/686). Characteristics of the two groups are displayed in Table 4.

Calculation of risk scores for all 686 patients revealed significantly higher scores in the abdominal wound dehiscence group $(P<0.001)$. Median scores were 5.7 in the abdominal wound dehiscence group (range: $2.8-8.5$ ) and 2.9 in the control group (range: 0-7.6). Logistic regression analysis of abdominal wound dehiscence in relation to the calculated risk scores showed that an increase of the risk score by one point is associated with an increase of the risk of abdominal wound dehiscence of $2.96(P<0.001)$. The fit of the model was good, as shown by the Hosmer and Lemeshow test $(P=0.79)$. The area under the curve in the ROC plot was 0.91 , showing a high predictive value of the risk score. The absolute risk of developing abdominal wound dehiscence in relation to the risk score is shown in Fig. 1, and the mean probability per risk score category is featured in Table 5.

The calculation of the probability of abdominal wound dehiscence for an individual surgical patient is performed in two steps. First, the total risk score is calculated by adding the weights of the various variables shown in Table 3. In the second step, the probability of developing abdominal wound dehiscence, $P$, is calculated according to the logistic formula:

$P=\mathrm{e}^{\mathrm{x}} /\left(1+\mathrm{e}^{x}\right) * 100 \%$,

where ' $\mathrm{e}$ ' represents ' $-8.37+(1.085 *$ calculated total risk score $)$ '.

For example, the risk score for a 67-year-old man who undergoes an elective reconstruction of the abdominal aorta and is known to have a history of chronic pulmonary disease is 0.9 (score for age 60-69 years) +0.7 (score for male gender) +1.3 (score for vascular surgery) +0.7 (score for chronic pulmonary disease), for a total of 3.6. The probability, $P$, of this patient's developing abdominal wound dehiscence is:

$\mathrm{e}^{(-8.37+(1.085 * 3.6))} / 1+\mathrm{e}^{(-8.37+(1.085 * 3.6))} * 100 \%=1.1 \%$.

An emergency repair in a similar patient with a ruptured aneurysm and subsequent anemia results in a total score of 4.9 (i.e., subtotal of 3.6 points +0.6 emergency +0.7 anemia). Thus, the absolute risk rises to $4.5 \%$. 
Table 1 Characteristics of the two groups in the study

\begin{tabular}{|c|c|c|c|c|}
\hline \multirow[t]{40}{*}{$\begin{array}{l}\text { Table } 1 \text { Characteristics of the } \\
\text { two groups in the study }\end{array}$} & Variable & $\begin{array}{l}\text { Abdominal wound } \\
\text { dehiscence }(n=363)\end{array}$ & $\begin{array}{l}\text { Control group } \\
(n=1,089)\end{array}$ & $P$ value \\
\hline & Age, years & $65 \pm 14.1(19-91)$ & $57 \pm 16.0(18-95)$ & $<0.001$ \\
\hline & $<40$ & $8 \%(28)$ & $21 \%(230)$ & \\
\hline & $40-49$ & $11 \%(39)$ & $16 \%(173)$ & \\
\hline & $50-59$ & $20 \%(71)$ & $21 \%(232)$ & \\
\hline & $60-69$ & $28 \%(102)$ & $24 \%(256)$ & \\
\hline & $>70$ & $34 \%(123)$ & $18 \%(198)$ & \\
\hline & Gender & & & \\
\hline & Male & $75 \%(272)$ & $56 \%(604)$ & $<0.001$ \\
\hline & Female & $25 \%(91)$ & $45 \%(485)$ & \\
\hline & Previous laparotomy & $46 \%(165)$ & $50 \%(540)$ & 0.173 \\
\hline & Hypertension & $46 \%(168)$ & $31 \%(332)$ & $<0.001$ \\
\hline & Diabetes mellitus & $9 \%(33)$ & $9 \%(101)$ & 0.917 \\
\hline & Chronic pulmonary disease & $29 \%(104)$ & $12 \%(129)$ & $<0.001$ \\
\hline & Corticosteroid use & $30 \%(109)$ & $18 \%(200)$ & $<0.001$ \\
\hline & Malignancy & & & \\
\hline & Local disease & $34 \%(122)$ & $20 \%(221)$ & $<0.001$ \\
\hline & Metastases & $13 \%(46)$ & $19 \%(204)$ & \\
\hline & Ascites & $23 \%(84)$ & $5 \%(59)$ & $<0.001$ \\
\hline & Jaundice & $15 \%(54)$ & $8 \%(307)$ & $<0.001$ \\
\hline & Anemia & $61 \%(223)$ & $35 \%(377)$ & $<0.001$ \\
\hline & Uremia & $31 \%(112)$ & $23 \%(245)$ & 0.013 \\
\hline & Sepsis & $20 \%(72)$ & $8 \%(83)$ & $<0.001$ \\
\hline & Emergency surgery & $46 \%(165)$ & $26 \%(285)$ & $<0.001$ \\
\hline & Type of surgery & & & \\
\hline & Abdominal wall & $21 \%(76)$ & $27 \%(296)$ & $<0.001$ \\
\hline & Gallbladder/bile duct & $5 \%(19)$ & $7 \%(79)$ & \\
\hline & Esophagus & $9 \%(32)$ & $6 \%(61)$ & \\
\hline & Gastroduodenal & $8 \%(28)$ & $5 \%(50)$ & \\
\hline & Small bowel & $7 \%(26)$ & $8 \%(90)$ & \\
\hline & Large bowel & $27 \%(98)$ & $19 \%(203)$ & \\
\hline & Vascular & $15 \%(54)$ & $10 \%(107)$ & \\
\hline & Kidney & $2 \%(7)$ & $7 \%(71)$ & \\
\hline & Liver & $4 \%(13)$ & $5 \%(56)$ & \\
\hline & Pancreas & $2 \%(6)$ & $5 \%(51)$ & \\
\hline & Adrenal gland & $0 \%(0)$ & $1 \%(9)$ & \\
\hline & Spleen & $1 \%(4)$ & $2 \%(16)$ & \\
\hline & Operative time (min) & $207 \pm 134(30-755)$ & $180 \pm 126(25-735)$ & 0.003 \\
\hline & $<150 \min$ & $32 \%(117)$ & $39 \%(425)$ & 0.024 \\
\hline & $\geq 150 \mathrm{~min}$ & $68 \%(246)$ & $61 \%(664)$ & \\
\hline \multirow{3}{*}{$\begin{array}{l}\text { Data are presented as } \\
\text { percentages, with numbers in } \\
\text { parentheses, or as mean } \pm \mathrm{SD} \\
\text { (range) }\end{array}$} & Coughing & $17 \%(46)$ & $4 \%(36)$ & $<0.001$ \\
\hline & Vomiting & $3 \%(6)$ & $3 \%(33)$ & 0.662 \\
\hline & Wound infection & $52 \%(188)$ & $11 \%(121)$ & $<0.001$ \\
\hline
\end{tabular}

Data are presented as percentages, with numbers in parentheses, or as mean $\pm \mathrm{SD}$ (range)

\section{Discussion}

In recent years, surgical therapy has become increasingly adjusted to individual patients based on their specific risk profiles. The goal of this strategy is to affect treatment outcomes positively. Furthermore, informed consent issues are gaining more attention from patient organizations, lawyers, and doctors in the light of juridical procedures. Before obtaining informed consent, patients should be fully informed about complications that can be expected to 
Table 2 Results of multivariate logistic regression analysis

\begin{tabular}{|c|c|c|c|c|c|}
\hline \multirow[t]{2}{*}{ Variable } & \multirow{2}{*}{$\begin{array}{l}\text { Regression } \\
\text { coefficient }\end{array}$} & \multirow{2}{*}{$\begin{array}{l}\text { Odds } \\
\text { ratio }(\mathrm{OR})\end{array}$} & \multicolumn{2}{|c|}{$95 \%$ Confidence Interval for OR } & \multirow[t]{2}{*}{$P$ value } \\
\hline & & & Lower limit & Upper limit & \\
\hline Age category $(\text { years })^{\mathrm{a}}$ & & & & & $0.002^{\mathrm{b}}$ \\
\hline $40-49$ & 0.43 & 1.54 & 0.81 & 2.93 & 0.192 \\
\hline $50-59$ & 0.89 & 2.44 & 1.37 & 4.34 & 0.002 \\
\hline $60-69$ & 0.89 & 2.43 & 1.39 & 4.26 & 0.002 \\
\hline$>70$ & 1.09 & 2.96 & 1.67 & 5.25 & $<0.001$ \\
\hline Male gender & 0.72 & 2.05 & 1.44 & 2.90 & $<0.001$ \\
\hline Chronic pulmonary disease & 0.72 & 2.05 & 1.39 & 3.01 & $<0.001$ \\
\hline Ascites & 1.49 & 4.43 & 2.68 & 7.33 & $<0.001$ \\
\hline Anemia & 0.72 & 2.05 & 1.48 & 2.84 & $<0.001$ \\
\hline Jaundice & 0.56 & 1.75 & 1.02 & 3.00 & 0.042 \\
\hline Emergency surgery & 0.59 & 1.80 & 1.27 & 2.55 & 0.001 \\
\hline Type of surgery ${ }^{c}$ & & & & & $<0.001^{\mathrm{b}}$ \\
\hline Gallbladder/bile duct & 0.70 & 2.02 & 0.93 & 4.37 & 0.075 \\
\hline Esophagus & 1.45 & 4.28 & 2.21 & 8.28 & $<0.001$ \\
\hline Gastroduodenum & 1.38 & 3.97 & 2.05 & 7.69 & $<0.001$ \\
\hline Small bowel & 0.94 & 2.55 & 1.32 & 4.90 & 0.005 \\
\hline Large bowel & 1.38 & 3.97 & 2.45 & 6.45 & $<0.001$ \\
\hline Vascular & 1.25 & 3.50 & 2.01 & 6.09 & $<0.001$ \\
\hline Kidney & -0.11 & 0.90 & 0.35 & 2.27 & 0.819 \\
\hline Liver & 0.11 & 1.12 & 0.46 & 2.74 & 0.804 \\
\hline Pancreas & -0.41 & 0.66 & 0.23 & 1.91 & 0.446 \\
\hline Other $^{\mathrm{d}}$ & 0.30 & 1.35 & 0.53 & 1.71 & 0.669 \\
\hline Coughing & 1.42 & 4.15 & 2.49 & 6.91 & $<0.001$ \\
\hline Wound infection & 1.86 & 6.43 & 4.56 & 9.06 & $<0.001$ \\
\hline
\end{tabular}

occur. Thus, preoperative risk assessment and information on absolute risk is important for both patients and doctors.

We have developed a risk model based on a large group of patients with abdominal wound dehiscence and compared possible risk factors with a large control group, all from a single academic teaching hospital. A risk model was designed based on the relative weights of the various risk factors. The model was validated in a separate population and demonstrated high predictive value for abdominal wound dehiscence, supporting the hypothesis that the variables identified as risk factors are actual risk factors. Calculation of the absolute risk, $P$, for a particular patient is performed by adding the weights of the various risk factors. The resulting risk score is subsequently entered into the given formula to obtain the absolute risk for that patient. The probability can also be deduced more easily from Fig. 1 by tracing the percentage that corresponds with the calculated risk score. This figure also shows that the probability of developing abdominal wound dehiscence increases exponentially with higher scores and more risk factors.

Although the risk model has shown high predictive value for abdominal wound dehiscence, the relative weight of the risk factors may differ slightly in reality. Our method of control group selection could have induced a bias concerning the relative weight of the factor "emergency surgery." For patients with abdominal wound dehiscence who were primarily treated during weekends and holidays, control patients were selected from patients operated on the following workdays. Therefore, the effect of emergency surgery might have been overestimated in our study. It has been reported though, to be a highly significant factor in other studies [4-6, 9, 11, 13, 16]. Patients who undergo emergency surgery are generally in worse condition and nutritional state, and the chance of contamination of the surgical field is higher than in elective surgery. Moreover, the performance of the surgeon might be affected at night, which could lead to suboptimal closure of the abdomen at the end of the operation.

Old age is another independent risk factor for abdominal wound dehiscence. Age has also been reported as a risk factor in other studies [6, 8-10, 12, 13, 15]. The explanation for this might lie in deterioration of the tissue repair mechanism in the elderly. Especially during the first few days of the wound healing process, the immune system plays a key role. Functional changes adversely affect the influx of cells and compounds that are essential for tissue repair [18]. Anemia is a risk factor that is related to increased perioperative stress, blood transfusions, and 
Table 3 Risk score for abdominal wound dehiscence
Theoretical score (min-max): $0-10.6$

\begin{tabular}{ll}
\hline Variable & $\begin{array}{l}\text { Risk } \\
\text { score }\end{array}$ \\
\hline Age category, years & \\
40-49 & 0.4 \\
$50-59$ & 0.9 \\
$60-69$ & 0.9 \\
$>70$ & 1.1 \\
Male gender & 0.7 \\
Chronic pulmonary & 0.7 \\
disease & \\
Ascites & 1.5 \\
Jaundice & 0.5 \\
Anemia & 0.7 \\
Emergency surgery & 0.6 \\
Type of surgery & \\
Gallbladder/bile duct & 0.7 \\
Esophagus & 1.5 \\
Gastroduodenum & 1.4 \\
Small bowel & 0.9 \\
Large bowel & 1.4 \\
Vascular & 1.3 \\
Coughing & 1.4 \\
Wound infection & 1.9 \\
\hline
\end{tabular}

decreased tissue oxygenation, all of which can affect the immune system and the wound healing process [19, 20].

One of the interesting risk factors found in this study, is gender. In previous studies, males have been reported to have a higher risk of developing abdominal wound dehiscence $[6-8,12]$. The reason for this disadvantage is not entirely clear. One of the possible confounders may be smoking. Because most smokers from the studied generations tended to be male, the effect of gender may be confounded with the effect of smoking on tissue repair. Unfortunately, smoking has thus far not been investigated as an independent risk factor for abdominal wound dehiscence. Because of the lack of sufficient data, this factor could not be investigated in the present study either. Another explanation may be that men build up higher abdominal wall tension than females. An increase in intraabdominal pressure results in higher strain on the wound edges, causing the sutures to cut through the muscles and fascia. This explanation may also apply to ascites and coughing, causing increment in intra-abdominal pressure.

In the present study, wound infection proved to be the risk factor with the highest relative weight. Its importance has been confirmed by virtually every study on this topic [4-7, 9, 12-15]. Continued presence of bacteria causes influx and activation of neutrophils and increases in levels of degradative matrix metalloproteinases (MMPs). In the absence of sufficient tissue inhibitors of MMPs, wound degradation will occur [21]. The release of endotoxins by
Table 4 Characteristics of the validation population

\begin{tabular}{|c|c|c|}
\hline Variable & $\begin{array}{l}\text { Abdominal wound } \\
\text { dehiscence } \\
(n=19)\end{array}$ & $\begin{array}{l}\text { No abdominal } \\
\text { wound dehiscence } \\
(n=667)\end{array}$ \\
\hline Age, years & $66 \pm 9.6(42-79)$ & $58 \pm 15.7(18-99)$ \\
\hline$<40$ & $0 \%(0)$ & $17 \%(111)$ \\
\hline $40-49$ & $11 \%(2)$ & $16 \%(106)$ \\
\hline $50-59$ & $11 \%(2)$ & $23 \%(155)$ \\
\hline $60-69$ & $37 \%(7)$ & $23 \%(156)$ \\
\hline$>70$ & $42 \%(8)$ & $21 \%(139)$ \\
\hline Male & $58 \%(11)$ & $56 \%(373)$ \\
\hline Female & $42 \%(8)$ & $44 \%(294)$ \\
\hline Chronic pulmonary disease & $16 \%(3)$ & $14 \%(96)$ \\
\hline Ascites & $26 \%(5)$ & $9 \%(57)$ \\
\hline Jaundice & $5 \%(1)$ & $5 \%(35)$ \\
\hline Anemia & $79 \%(15)$ & $38 \%(255)$ \\
\hline Emergency surgery & $47 \%(9)$ & $37 \%(248)$ \\
\hline Abdominal wall & $5 \%(1)$ & $13 \%(84)$ \\
\hline Gallbladder/bile duct & $0 \%(0)$ & $6 \%(38)$ \\
\hline Gastroduodenum & $16 \%(3)$ & $7 \%(44)$ \\
\hline Small bowel & $5 \%(1)$ & $8 \%(50)$ \\
\hline Large bowel & $37 \%(7)$ & $19 \%(127)$ \\
\hline Vascular & $11 \%(2)$ & $6 \%(41)$ \\
\hline Esophagus & $5 \%(1)$ & $8 \%(56)$ \\
\hline Adrenal gland & $0 \%(0)$ & $0 \%(0)$ \\
\hline Kidney & $11 \%(2)$ & $19 \%(124)$ \\
\hline Liver & $5 \%(1)$ & $11 \%(72)$ \\
\hline Pancreas & $5 \%(1)$ & $4 \%(24)$ \\
\hline Spleen & $0 \%(0)$ & $1 \%(6)$ \\
\hline Coughing & $32 \%(6)$ & $11 \%(75)$ \\
\hline Wound infection & $90 \%(17)$ & $17 \%(112)$ \\
\hline
\end{tabular}

Data are presented as percentages, with numbers between parentheses, or as mean $\pm \mathrm{SD}$ (range)

bacteria leads to the production of collagenase, which degrades collagen fibers [22]. Infection thereby causes a prolongation of the inflammatory phase and negatively affects deposition of collagen and fibroblast activity. In wounds of patients with abdominal wound dehiscence, it has been observed that degradation of collagen exceeds the synthesis of collagen, which adversely affects breaking strength [23]. Adequate tissue breaking strength is necessary, however, to provide support for the sutures that hold the wound edges together. Low breaking strength can therefore amount to abdominal wound dehiscence, especially in the presence of increased intra-abdominal pressure and abnormal inflammatory response [18]. Primary repair can be difficult or impossible when tissue has low breaking strength, creating the need for the use of mesh or acceptance of the high risk of recurrent abdominal wound dehiscence.

Risk factors that did not have independent effects in our evaluation included hypertension, uremia, and corticosteroid 


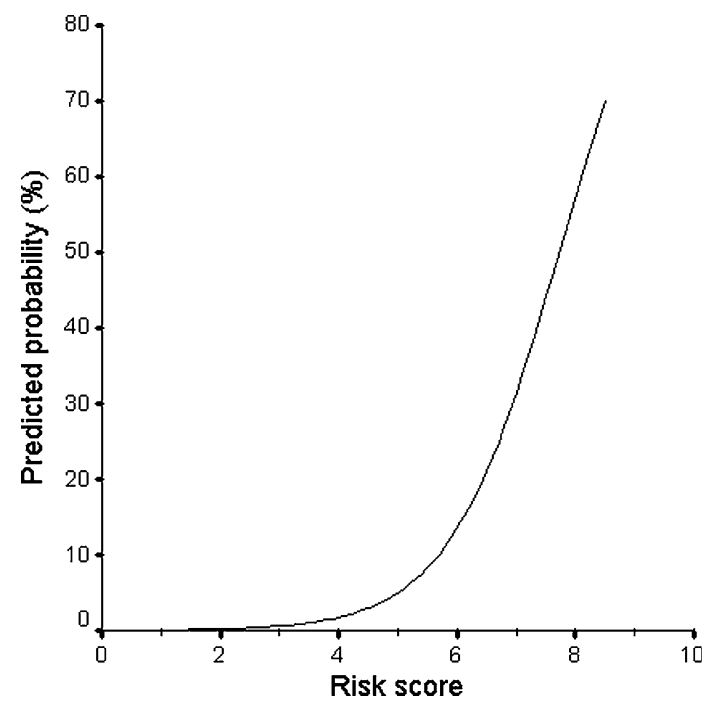

Fig. 1 Predicted probability (\%) of developing abdominal wound dehiscence according to risk score

use, although these factors have been described as risk factors by a number of authors $[9,13,15,24]$. The latter can be explained by the more frequent use of corticosteroids in lung disease patients, which applied to both cases and controls in our study. We found no significant effect on the occurrence of abdominal wound dehiscence for diabetes mellitus and previous laparotomy. Malignancy, sepsis, and postoperative vomiting have been identified as risk factors by several authors, but no significant effects were found in the present study $[7,9,11,13-15]$. This was surprising because it was suspected that the presence of scar tissue, microvascular changes due to hypertension and diabetes, poor tissue perfusion, and poor overall condition of the patient, associated with sepsis and malignancy, would be risk factors. Jaundice, on the other hand, was found to be an independent risk factor. This has not been confirmed by other studies [7, 11-15]. Most important, Armstrong investigated jaundice in relation to hematocrit and albumin levels and malignancy [7]. Jaundice was significant in univariate analysis but not in multivariate analysis in that study. The conclusion of that study was that wound healing is affected in jaundiced patients due to the association with low hematocrit and albumin levels and malignancy (i.e., poor nutritional status) and not to raised bilirubin levels. Low protein and albumin levels and deficiencies of several vitamins and minerals such as vitamins $\mathrm{A}, \mathrm{B}_{1}, \mathrm{~B}_{2}, \mathrm{~B}_{6}, \mathrm{C}$ and zinc and copper have been associated with poor wound repair [18]. Data on preoperative albumin levels were available for $83 \%$ of patients with abdominal wound dehiscence and $56 \%$ of controls. Albumin levels were below $35 \mathrm{~g} / 1$ in $63 \%$ of patients with abdominal wound dehiscence and $34 \%$ of controls, which was significantly less $(P<0.001)$ and suggestive of an association between low albumin levels and development of abdominal wound dehiscence.

Additional investigation is needed to determine the value of the underlying risk score in other settings. Also, studies are needed to evaluate other possible factors for which limited retrospective data are available, such as nutritional state. The consequences of the score are also limited by the inclusion of risk factors that occur in the postoperative phase, such as coughing and wound infection. Still, because the model has been shown to be highly predictive, it can be used to identify patients at risk. Preventive measures, e.g., the use of mesh and special suture techniques and materials, aimed at decreasing tension on the wound edges, can be investigated and used in these patient groups. Tohme et al., for example, reported the results of a retrospective study on the preventive use of polyglactin 910 mesh versus retention sutures in patients with at least one suspected risk factor for abdominal wound dehiscence [25]. These factors included malnutrition with loss of over $10 \%$ of body weight, obesity, cirrhosis, and/or ascites, neoplastic diseases, immune depression due to corticosteroid use or chemotherapy, chronic respiratory insufficiency, repeated intervention, and diffuse or local peritonitis. Although the incidence of abdominal wound dehiscence was significantly lower in the polyglactin 910 mesh group (0/66 versus $14 / 226$ patients, $P<0.05$ ), no stratification was made for the predicted risk of abdominal wound dehiscence, which

Table 5 Absolute risk of abdominal wound dehiscence in the validation population by risk score

\begin{tabular}{lccccc}
\hline Risk score & Total no. & Percent & \multicolumn{2}{l}{ Abdominal wound dehiscence } & \multirow{2}{*}{ Mean probability ${ }^{\mathrm{b}}(\%)$} \\
\cline { 5 - 6 } & & & Number & Percent \\
\hline $0-2$ & 188 & 27.4 & 0 & 0.0 & 0.1 \\
$2-4$ & 329 & 48.0 & 2 & 0.6 & 0.7 \\
$4-6$ & 138 & 20.1 & 8 & 5.8 & 5.5 \\
$6-8$ & 29 & 4.2 & 7 & 24.1 & 26.2 \\
$>8$ & 2 & 0.3 & 2 & 100 & 66.5 \\
Total & 686 & 100 & 19 & 2.8 & \\
\hline
\end{tabular}

a Observed percentages within risk score groups

b Mean values of individual calculated probabilities according to risk score within risk score group 
hampers the interpretation and extrapolation of the results of this study. The same holds true for other future studies on closure technique with abdominal wound dehiscence as study outcome. The necessity of good surgical technique is underlined by the fact that broken sutures and loose knots accounted for $12 \%$ of the cases of abdominal wound dehiscence in these series. We therefore hope that the results of this study will lead to better, evidence-based treatment options for abdominal wound dehiscence and, eventually, a lower incidence of this severe complication.

In conclusion, various putative risk factors for abdominal wound dehiscence were investigated in the thus far largest study in the general surgical population. Important risk factors for abdominal wound dehiscence have been identified in this case-control study, including age, gender, chronic pulmonary disease, ascites, jaundice, anemia, emergency surgery, type of surgery, coughing, and wound infection. On the basis of these data, we were able to develop a risk score for abdominal wound dehiscence. This score can be entered into a formula to calculate the probability of developing abdominal wound dehiscence for individual patients. High-risk patients, for instance with scores of 6 or higher without counting postoperative factors such as coughing and wound infection, have a probability of developing abdominal wound dehiscence of more than $13.5 \%$. This type of patient would be interesting to include in future intervention studies that could involve preventive wound closing with such reinforcements as (biologic) mesh. Furthermore, utmost efforts should be made to consider minimally invasive surgery, also if other centers need to be involved for this indication. The risk model has shown high predictive value for the occurrence of this severe complication in the validation analyses. From the results of this study, we can also conclude that a number of risk factors for abdominal wound dehiscence can be mitigated during the perioperative period. This implies that the risk of developing abdominal wound dehiscence can be reduced by preventing pneumonia and wound infection, and by applying optimal surgical technique in every patient.

Acknowledgments The authors are grateful for the support provided by the employees of the Central Medical Archives, who retrieved patient data and charts.

Open Access This article is distributed under the terms of the Creative Commons Attribution Noncommercial License which permits any noncommercial use, distribution, and reproduction in any medium, provided the original author(s) and source are credited.

\section{References}

1. Fleischer GM, Rennert A, Rühmer M (2000) Die infizierte Bauchdecke und der Platzbauch. Chirurg 71:754-762
2. Poole GV Jr (1985) Mechanical factors in abdominal wound closure: the prevention of fascial dehiscence. Surgery 97:631640

3. Carlson MA (1997) Acute wound failure. Surg Clin North Am 77:607-636

4. Webster C, Neumayer L, Smout R et al (2003) National Veterans Affairs Surgical Quality Improvement Program. Prognostic models of abdominal wound dehiscence after laparotomy. J Surg Res 109:130-137

5. Wahl W, Menke H, Schnütgen M et al (1992) Die Fasciendehiscenz-Ursache und Prognose. Chirurg 63:666-671

6. Gislason H, Grønbech JE, Søreide O (1995) Burst abdomen and incisional hernia after major gastrointestinal operations-comparison of three closure techniques. Eur J Surg 161:349-354

7. Armstrong CP, Dixon JM, Duffy SW et al (1984) Wound healing in obstructive jaundice. Br J Surg 71:267-270

8. Penninckx FM, Poelmans SV, Kerremans RP et al (1979) Abdominal wound dehiscence in gastroenterological surgery. Ann Surg 189:345-352

9. Pavlidis TE, Galatianos IN, Papaziogas BT et al (2001) Complete dehiscence of the abdominal wound and incriminating factors. Eur J Surg 167:351-354

10. Humar A, Ramcharan T, Denny R et al (2001) Are wound complications after a kidney transplant more common with modern immunosuppression? Transplantation 72:1920-1923

11. Mäkelä JT, Kiviniemi H, Juvonen T et al (1995) Factors influencing wound dehiscence after midline laparotomy. Am J Surg 170:387-390

12. Keill RH, Keitzer WF, Nichols WK et al (1973) Abdominal wound dehiscence. Arch Surg 106:573-577

13. Niggebrugge AH, Hansen BE, Trimbos JB et al (1995) Mechanical factors influencing the incidence of burst abdomen. Eur J Surg 161:655-661

14. Cöl C, Soran A, Cöl M (1998) Can postoperative abdominal wound dehiscence be predicted? Tokai J Exp Clin Med 23:123127

15. Riou JP, Cohen JR, Johnson H Jr (1992) Factors influencing wound dehiscence. Am J Surg 163:324-330

16. Swaroop M, Williams M, Greene WR et al (2005) Multiple laparotomies are a predictor of fascial dehiscence in the setting of severe trauma. Am Surg 71:402-405

17. Halasz NA (1968) Dehiscence of laparotomy wounds. Am J Surg 116:210-214

18. Dubay DA, Franz MG (2003) Acute wound healing: the biology of acute wound failure. Surg Clin North Am 83:463-481

19. Carson JL, Duff A, Poses RM et al (1996) Effect of anaemia and cardiovascular disease on surgical mortality and morbidity. Lancet 348:1055-1060

20. Carson JL, Noveck H, Berlin JA et al (2002) Mortality and morbidity in patients with very low postoperative $\mathrm{Hb}$ levels who decline blood transfusion. Transfusion 42:812-818

21. Menke NB, Ward KR, Witten TM et al (2007) Impaired wound healing. Clin Dermatol 25:19-25

22. Broughton G II, Janis JE, Attinger CE (2006) Wound healing: an overview. Plast Reconstr Surg 177:1e-S-32e-S

23. Renvall S, Grönroos I, Laato M (2001) Burst abdomen. Local synthesis of nucleic acids, glycosaminoglycans, proteins and collagen in wounds. Ann Chir Gynaecol 90(Suppl 215):33-37

24. Niggebrugge AH, Trimbos JB, Hermans J et al (1999) Influence of abdominal-wound closure technique on complications after surgery: a randomised study. Lancet 353(9164):1563-1567

25. Tohme C, Brechet E, Bernard A et al (1991) Prévention de l'éviscération post-opératoire. Ann Chir 45:513-516 